\title{
ON SPECTRUM OF A GENERAL PETROVSKY TYPE EQUATION AND RIESZ BASIS OF $N$-CONNECTED BEAMS WITH LINEAR FEEDBACK AT JOINTS
}

\author{
BAO-ZHU GUO, YU XIE, and XUE-ZHANG HOU
}

\begin{abstract}
A framework of a general type of Petrovsky equation is formulated. The characteristic equation for eigenvalues of the system is derived and the associated eigenfunctions are found. For $\mathrm{N}$ connected beams with linear feedbacks at joint points, the asymptotic expansions of eigenvalues and eigenfunctions are further developed, and the Riesz basis property and the exponential stability are then concluded.
\end{abstract}

\section{INTRODUCTION}

The vibration and control of serially connected strings and EulerBernoulli beams with linear feedback controls at joints have been studied extensively in the last two decades (see, e.g., $[2-4,11,16,17,20,21])$. In addition to the analysis of the distribution of eigenvalues, one also needs to establish the so-called spectrum-determined growth condition in order to conclude exponential stability for these infinite-dimensional systems from spectral analysis. In the case of serially connected strings, the first results on exponential stability were obtained in [16] for a 2-connected strings with linear feedbacks at the middle of the span. The stability of $N$-connected strings under joints feedbacks was studied in [17]. It is shown in [19] that any system of $N$-connected strings with linear feedbacks at the joint points can be put into a first-order homogeneous hyperbolic equation in the following general form:

$$
\left\{\begin{array}{l}
\frac{\partial}{\partial t}\left[\begin{array}{l}
\boldsymbol{u}(x, t) \\
\boldsymbol{v}(x, t)
\end{array}\right]=K(x) \frac{\partial}{\partial x}\left[\begin{array}{l}
\boldsymbol{u}(x, t) \\
\boldsymbol{v}(x, t)
\end{array}\right], \quad x \in(0,1), \quad t>0 \\
\boldsymbol{v}(1, t)=D \boldsymbol{u}(1, t), \quad \boldsymbol{u}(0, t)=E \boldsymbol{v}(0, t),
\end{array}\right.
$$

where $K(x)=\operatorname{diag}\left\{\lambda_{1}(x), \ldots, \lambda_{N}(x), \mu_{N+1}(x), \ldots, \mu_{n}(x)\right\}$ is a diagonal $(n \times n)$-matrix with real entries $\lambda_{i}(x) \in C^{1}[0,1], \mu_{j}(x) \in C^{1}[0,1]$ and

2000 Mathematics Subject Classification. 93C20, 93D15, 35B35, 35P10.

Key words and phrases. Petrovsky type equation, $C_{0}$-semigroup, spectral analysis, Riesz basis. 
$\lambda_{i}(x)>0, \mu_{j}(x)<0$ for all $x \in[0,1], i=1,2, \ldots, N, j=N+1, \ldots, n$,

$$
\begin{gathered}
\boldsymbol{u}(x, t)=\left(u_{1}(x, t), u_{2}(x, t), \ldots, u_{N}(x, t)\right)^{T}, \\
\boldsymbol{v}(x, t)=\left(v_{N+1}(x, t), v_{N+2}(x, t), \ldots, v_{n}(x, t)\right)^{T}
\end{gathered}
$$

are column vectors in $\mathbb{R}^{N}$ and $\mathbb{R}^{n-N}$, respectively, and $D$ and $E$ are real constant matrices of appropriate sizes. It was proved in [14] that the spectrumdetermined growth condition always holds for system (1.1).

For Euler-Bernoulli beams, using the frequency approach, exponential stability was studied for a single beam equation with moment boundary feedback control due to failure of finding the energy multiplier which is one of the main approaches in proving the exponential stability of the system [5] and for 2-connected beams [21] under linear feedback control at dissipative joints. On the other hand, the spectrum analysis was carried out for 2connected beams [3] and for general $N$-connected beams [20] under joint linear feedback controls. It turned out to be very difficult to establish the spectrum-determined growth condition for distributed parameter systems [22]. Euler-Bernoulli beams are not an exception. Even for a single beam equation, the proof of the exponential stability is difficult despite that its spectral distribution is clear (see, e.g., [5]).

Recently, an alternative referred to as the Riesz basis approach was proposed which may lead to a more profound result than the stability analysis. In this approach, instead of the spectrum-determined growth condition, one tries to establish the Riesz basis property for the system. If successful, the growth condition can then be concluded as a consequence of existence of the Riesz basis. The Riesz basis for single beam equations was developed in [6-8]. The basis property for 2-connected beams was studied in $[9,10]$. In this paper, we study the following general Petrovsky type system [15] in one space variable in normal form:

$$
\left\{\begin{array}{l}
\frac{\partial}{\partial t}\left[\begin{array}{l}
\boldsymbol{u}(x, t) \\
\boldsymbol{v}(x, t)
\end{array}\right]=K \frac{\partial^{2}}{\partial x^{2}}\left[\begin{array}{l}
\boldsymbol{u}(x, t) \\
\boldsymbol{v}(x, t)
\end{array}\right], \quad x \in(0,1), \quad t>0, \\
{[A, B]\left[\boldsymbol{u}_{x}(0, t), \boldsymbol{v}_{x}(0, t), \boldsymbol{u}(0, t), \boldsymbol{v}(0, t)\right]^{T}=0,} \\
{[E, F]\left[\boldsymbol{u}_{x}(1, t), \boldsymbol{v}_{x}(1, t), \boldsymbol{u}(1, t), \boldsymbol{v}(1, t)\right]^{T}=0,}
\end{array}\right.
$$

where

$$
\begin{aligned}
\boldsymbol{u}(x, t) & =\left[u_{1}(x, t), u_{2}(x, t), \ldots, u_{n}(x, t)\right]^{T}, \\
\boldsymbol{v}(x, t) & =\left[v_{1}(x, t), v_{2}(x, t), \ldots, v_{n}(x, t)\right]^{T}
\end{aligned}
$$


are column vectors in $\mathbb{R}^{n}, \boldsymbol{u}_{x}$ and $\boldsymbol{v}_{x}$ denote the derivatives of $\boldsymbol{u}$ and $\boldsymbol{v}$ with respect to $x$, respectively; $A, B, E$, and $F$ are real, constant $(2 n \times 2 n)$ matrices; $K$ is a constant $(2 n \times 2 n)$-matrix of the form

$$
\begin{gathered}
K=\left[\begin{array}{cc}
0 & \Upsilon \\
-\Upsilon & 0
\end{array}\right], \\
\Upsilon=\operatorname{diag}\left[l_{1}^{-2},-l_{2}^{-2}, \ldots,(-1)^{n+1} l_{n}^{-2}\right], \quad l_{j}>0, \quad i=1,2, \ldots, n .
\end{gathered}
$$

The contribution of this paper is as follows:

(a) a general approach is presented for the analysis of the distribution of eigenvalues of system (1.2);

(b) the asymptotics of eigenpairs of an $N$-connected beam equation with linear joints are obtained;

(c) the Riesz basis property is proved for the $N$-connected beam system.

The paper is organized as follows. In the next section, the characteristic equation of system (1.2) is derived and the eigenfunctions are found. The general treatment of the asymptotic expansions of the eigenvalues is presented in Sec. 3. Section 4 is devoted to a system of $N$-connected beams. Riesz basis for this special system is obtained in Sec. 5. The exponential stability is proved in Sec. 6. Finally, in Sec. 7, we give some remarks on some unsolved problems.

\section{Characteristic equation}

In this section, we derive the characteristic equation satisfied by eigenvalues of system (1.2). To begin with, we put system (1.2) into the framework of evolutionary equations in an underlying Hilbert space $\mathcal{H}$. Take $\mathcal{H}=\left(L^{2}(0,1)\right)^{2 n}$ and define $\mathcal{A}: D(\mathcal{A})(\subset \mathcal{H}) \rightarrow \mathcal{H}$ by

$$
\mathcal{A}\left[\begin{array}{l}
\boldsymbol{u} \\
\boldsymbol{v}
\end{array}\right]=K \frac{\partial^{2}}{\partial x^{2}}\left[\begin{array}{l}
\boldsymbol{u} \\
\boldsymbol{v}
\end{array}\right]
$$

where

$$
D(\mathcal{A})=\left\{\begin{array}{l|l}
{[\boldsymbol{u}, \boldsymbol{v}]^{T} \in\left(H^{2}(0,1)\right)^{2 n} \mid \begin{array}{l}
{[A, B]\left[\boldsymbol{u}_{x}(0), \boldsymbol{v}_{x}(0), \boldsymbol{u}(0), \boldsymbol{v}(0)\right]^{T}=0,} \\
{[E, F]\left[\boldsymbol{u}_{x}(1), \boldsymbol{v}_{x}(1), \boldsymbol{u}(1), \boldsymbol{v}(1)\right]^{T}=0}
\end{array}}
\end{array}\right\}
$$

and $H^{2}(0,1)$ denotes the usual Sobolev space. With this setting, system (1.2) can be considered as an abstract equation in $\mathcal{H}$ :

$$
\frac{d}{d t}\left[\begin{array}{l}
\boldsymbol{u} \\
\boldsymbol{v}
\end{array}\right]=\mathcal{A}\left[\begin{array}{l}
\boldsymbol{u} \\
\boldsymbol{v}
\end{array}\right] .
$$

Obviously, $\mathcal{A}$ is densely defined in $\mathcal{H}$. Next, we consider the eigenvalue problem for $\mathcal{A}$. For any given $\Phi=[\boldsymbol{f}, \boldsymbol{g}]^{T} \in \mathcal{H}$, solve the following equation:

$$
(\lambda-\mathcal{A})\left[\begin{array}{l}
\boldsymbol{u} \\
\boldsymbol{v}
\end{array}\right]=\left[\begin{array}{l}
\boldsymbol{f} \\
\boldsymbol{g}
\end{array}\right]
$$


i.e.,

$$
\left\{\begin{array}{l}
\frac{\partial^{2}}{\partial x^{2}}\left[\begin{array}{l}
\boldsymbol{u} \\
\boldsymbol{v}
\end{array}\right]=\lambda K^{-1}\left[\begin{array}{l}
\boldsymbol{u} \\
\boldsymbol{v}
\end{array}\right]-K^{-1} \Phi, \\
{[A, B]\left[\boldsymbol{u}_{x}(0), \boldsymbol{v}_{x}(0), \boldsymbol{u}(0), \boldsymbol{v}(0)\right]^{T}=0,} \\
{[E, F]\left[\boldsymbol{u}_{x}(1), \boldsymbol{v}_{x}(1), \boldsymbol{u}(1), \boldsymbol{v}(1)\right]^{T}=0,}
\end{array}\right.
$$

which can be further written as a first-order ordinary differential equation of the following form:

$$
\left\{\begin{array}{l}
\frac{\partial}{\partial x}\left[\begin{array}{c}
\boldsymbol{u}_{x} \\
\boldsymbol{v}_{x} \\
\boldsymbol{u} \\
\boldsymbol{v}
\end{array}\right]=\left[\begin{array}{cc}
0_{2 n} & \lambda K^{-1} \\
I_{2 n} & 0_{2 n}
\end{array}\right]\left[\begin{array}{c}
\boldsymbol{u}_{x} \\
\boldsymbol{v}_{x} \\
\boldsymbol{u} \\
\boldsymbol{v}
\end{array}\right]-\left[\begin{array}{c}
K^{-1} \Phi \\
0
\end{array}\right], \\
{[A, B]\left[\boldsymbol{u}_{x}(0), \boldsymbol{v}_{x}(0), \boldsymbol{u}(0), \boldsymbol{v}(0)\right]^{T}=0} \\
{[E, F]\left[\boldsymbol{u}_{x}(1), \boldsymbol{v}_{x}(1), \boldsymbol{u}(1), \boldsymbol{v}(1)\right]^{T}=0,}
\end{array}\right.
$$

where $I_{2 n}$ denotes the $2 n \times 2 n$ identity matrix. Set

$$
K_{\lambda}=\left[\begin{array}{cc}
0_{2 n} & \lambda K^{-1} \\
I_{2 n} & 0_{2 n}
\end{array}\right]
$$

Then the solution to the governing equation of (2.5) is

$$
\left[\begin{array}{c}
\boldsymbol{u}_{x}(x) \\
\boldsymbol{v}_{x}(x) \\
\boldsymbol{u}(x) \\
\boldsymbol{v}(x)
\end{array}\right]=e^{K_{\lambda} x}\left[\begin{array}{c}
\boldsymbol{u}_{x}(0) \\
\boldsymbol{v}_{x}(0) \\
\boldsymbol{u}(0) \\
\boldsymbol{v}(0)
\end{array}\right]-\int_{0}^{x} e^{K_{\lambda}(x-s)}\left[\begin{array}{c}
K^{-1} \Phi \\
0
\end{array}\right] d s .
$$

In order for (2.7) to satisfy (2.5), the last two boundary conditions should be fulfilled, i.e.,

$$
\left\{\begin{array}{l}
{[A, B]\left[\boldsymbol{u}_{x}(0), \boldsymbol{v}_{x}(0), \boldsymbol{u}(0), \boldsymbol{v}(0)\right]^{T}=0} \\
{[E, F] e^{K_{\lambda}}\left[\boldsymbol{u}_{x}(0), \boldsymbol{v}_{x}(0), \boldsymbol{u}(0), \boldsymbol{v}(0)\right]^{T}} \\
=\int_{0}^{1}[E, F] e^{K_{\lambda}(1-s)}\left[K^{-1} \Phi, 0\right]^{T} d s
\end{array}\right.
$$

Define

$$
H(\lambda)=\left[\begin{array}{c}
{[A, B]} \\
{[E, F] e^{K_{\lambda}}}
\end{array}\right]
$$

Then for

$$
h(\lambda)=\operatorname{det} H(\lambda) \neq 0
$$


it has

$$
\begin{aligned}
R(\lambda, \mathcal{A}) \Phi & =\left[0_{2 n}, I_{2 n}\right] e^{K_{\lambda} x}\left[\begin{array}{c}
\boldsymbol{u}_{x}(0) \\
\boldsymbol{v}_{x}(0) \\
\boldsymbol{u}(0) \\
\boldsymbol{v}(0)
\end{array}\right] \\
& -\int_{0}^{x}\left[0_{2 n}, I_{2 n}\right] e^{K_{\lambda}(x-s)}\left[\begin{array}{c}
K^{-1} \Phi \\
0
\end{array}\right] d s,
\end{aligned}
$$

where

$$
\left[\begin{array}{c}
\boldsymbol{u}_{x}(0) \\
\boldsymbol{v}_{x}(0) \\
\boldsymbol{u}(0) \\
\boldsymbol{v}(0)
\end{array}\right]=H^{-1}(\lambda)\left[\int_{0}^{1}[E, F] e^{K_{\lambda}(1-s)}\left[\begin{array}{c}
K^{-1} \Phi \\
0
\end{array}\right] d s\right] .
$$

Therefore, in this case, $\lambda \in \rho(\mathcal{A})$ and $R(\lambda, \mathcal{A})$ is compact.

On the other hand, if $h(\lambda)=0$, for any $4 n \times 1$ nonzero column vector $Z=\left(\boldsymbol{u}_{x}(0), \boldsymbol{v}_{x}(0), \boldsymbol{u}(0), \boldsymbol{v}(0)\right)^{T}$ satisfying $H(\lambda) Z=0$, by setting $\Phi=0$ in (2.7), we have

$$
\left[\begin{array}{c}
\boldsymbol{u}_{x}(x) \\
\boldsymbol{v}_{x}(x) \\
\boldsymbol{u}(x) \\
\boldsymbol{v}(x)
\end{array}\right]=e^{K_{\lambda} x} Z \neq 0
$$

and hence $\left(\boldsymbol{u}_{x}(x), \boldsymbol{v}_{x}(x), \boldsymbol{u}(x), \boldsymbol{v}(x)\right)^{T} \neq 0$. Therefore,

$$
\left[\begin{array}{l}
\boldsymbol{u} \\
\boldsymbol{v}
\end{array}\right]=\left[0_{2 n}, I_{2 n}\right]\left[\begin{array}{l}
\boldsymbol{u}_{x} \\
\boldsymbol{v}_{x} \\
\boldsymbol{u} \\
\boldsymbol{v}
\end{array}\right]=\left[0_{2 n}, I_{2 n}\right] e^{K_{\lambda} x} Z \neq 0
$$

and satisfies

$$
\mathcal{A}\left[\begin{array}{l}
\boldsymbol{u} \\
\boldsymbol{v}
\end{array}\right]=\lambda\left[\begin{array}{l}
\boldsymbol{u} \\
\boldsymbol{v}
\end{array}\right] .
$$

In other words, $\lambda \in \sigma(\mathcal{A})=\sigma_{p}(\mathcal{A})$.

Summarizing, we have obtained the following Theorem 1.

Theorem 1. Let $h(\lambda)=\operatorname{det} H(\lambda)$ be defined by $(2.10)$. Then $h(\lambda)$ is an entire function of $\lambda$, and the following statements hold:

(i) $\lambda \in \sigma(\mathcal{A})$ if and only if $h(\lambda)=0$, i.e.,

$$
\sigma(\mathcal{A})=\{\lambda \mid h(\lambda)=0\} .
$$

The eigenvalues are symmetric with respect to the real axis.

(ii) For each $\lambda \in \sigma(\mathcal{A})$, the corresponding eigenfunction $[\boldsymbol{u}, \boldsymbol{v}]^{T}$ is given by (2.13), where $Z$ is any nonzero solution of the algebraic equation $H(\lambda) Z=0$.

(iii) $\mathcal{A}$ is a densely defined discrete operator in $\mathcal{H}$, i.e., $\mathcal{A}$ is densely defined in $\mathcal{H}$ and $R(\lambda, \mathcal{A})=(\lambda-\mathcal{A})^{-1}$ is compact for any $\lambda \in \sigma(\mathcal{A})$. 
(iv) If $\mathcal{A}$ is dissipative, then $\mathcal{A}$ generates a $C_{0}$-semigroup of contractions on $\mathcal{H}$.

Proof. Items (i)-(iii) follow from the previous discussion, and (iv) follows from the Lumer-Phillips theorem in semigroup theory of linear operators (see, e.g., [1]).

\section{Asymptotic EXPANSion of Eigenvalues}

In this section, we always assume that $\mathcal{A}$ is dissipative. Under this assumption, all eigenvalues of $\mathcal{A}$ are located on the left complex half-plane with conjugate pairs. Let $\lambda=\rho^{2} \in \sigma(\mathcal{A})$. We may consider only $\lambda$ with $\pi / 2 \leq \arg (\lambda) \leq \pi$ owing to the symmetry of the distribution of eigenvalues. For these $\lambda$, we have

$$
\frac{\pi}{4} \leq \arg (\rho) \leq \frac{\pi}{2}
$$

Proposition 1. Assume that (3.1) holds. We set

$$
e^{2 \omega_{1} \rho}=y, \quad \omega_{1}=\frac{i+1}{\sqrt{2}} .
$$

Then $y$ satisfies

$$
f(y)+O\left(\rho^{-1}\right)=0, \quad|\rho| \rightarrow \infty,
$$

where $f(y)=\sum_{i=1}^{M} a_{i} y^{\xi_{i}}$ for some reals $a_{i}$ and $\xi_{i}$ and integer $M$.

Proof. Setting

$$
\begin{gathered}
\omega_{1}=\exp \left(\frac{\pi}{4 i}\right)=\frac{i+1}{\sqrt{2}}, \quad \omega_{2}=\exp \left(\frac{3 \pi}{4 i}\right)=\frac{i-1}{\sqrt{2}}, \\
\omega_{3}=-\omega_{1}, \quad \omega_{4}=-\omega_{2},
\end{gathered}
$$

which are the fourth-roots-of-unity of $x^{4}+1=0$, we have

$$
\left\{\begin{aligned}
\operatorname{Re}\left(\rho \omega_{1}\right) & =|\rho| \cos \left(\arg (\rho)+\frac{\pi}{4}\right) \leq 0, \\
\operatorname{Re}\left(\rho \omega_{2}\right) & =|\rho| \cos \left(\arg (\rho)+\frac{3}{4} \pi\right) \\
& =-|\rho| \sin \left(\arg (\rho)+\frac{\pi}{4}\right) \leq-|\rho| \cos \left(\frac{\pi}{4}\right) .
\end{aligned}\right.
$$

Now consider the eigenvalue problem $\mathcal{A}[\boldsymbol{u}, \boldsymbol{v}]^{T}=\lambda[\boldsymbol{u}, \boldsymbol{v}]^{T}$ in a different way as compared with (2.13):

$$
\left\{\begin{array}{l}
\frac{\partial^{2}}{\partial x^{2}}\left[\begin{array}{l}
\boldsymbol{u} \\
\boldsymbol{v}
\end{array}\right]=\lambda K^{-1}\left[\begin{array}{l}
\boldsymbol{u} \\
\boldsymbol{v}
\end{array}\right]=-\lambda \widetilde{K}\left[\begin{array}{l}
\boldsymbol{u} \\
\boldsymbol{v}
\end{array}\right], \\
{[A, B]\left[\boldsymbol{u}_{x}(0), \boldsymbol{v}_{x}(0), \boldsymbol{u}(0), \boldsymbol{v}(0)\right]^{T}=0,} \\
{[E, F]\left[\boldsymbol{u}_{x}(1), \boldsymbol{v}_{x}(1), \boldsymbol{u}(1), \boldsymbol{v}(1)\right]^{T}=0,}
\end{array}\right.
$$


where

$$
\begin{gathered}
T=\left[u_{1}, u_{2}, \ldots, u_{n}, v_{1}, v_{2}, \ldots, v_{n}\right]^{T}, \\
\widetilde{K}=\left[\begin{array}{cc}
0_{n} & \widetilde{\Upsilon} \\
-\widetilde{\Upsilon} & 0_{n}
\end{array}\right], \quad \widetilde{\Upsilon}=\operatorname{diag}\left[l_{1}^{2},-l_{2}^{2}, \ldots,(-1)^{n+1} l_{n}^{2}\right] .
\end{gathered}
$$

Let

$$
\left[\begin{array}{l}
\boldsymbol{u} \\
\boldsymbol{v}
\end{array}\right]=S Y(x), \quad S=\left[\begin{array}{cc}
I_{n} & I_{n} \\
-i P & i P
\end{array}\right], \quad S^{-1}=\frac{1}{2}\left[\begin{array}{cc}
I_{n} & i P \\
I_{n} & -i P
\end{array}\right],
$$

where $P=\operatorname{diag}\left[1,-1, \ldots,(-1)^{n+1}\right]$. Then

$$
Y^{\prime \prime}(x)=-\lambda S^{-1} \widetilde{K} S Y(x)
$$

and $-S^{-1} \widetilde{K} S$ is a diagonal matrix,

$$
-S^{-1} \widetilde{K} S=\left[\begin{array}{cc}
i \Lambda^{2} & 0_{n} \\
0_{n} & -i \Lambda^{2}
\end{array}\right], \quad \Lambda=\operatorname{diag}\left[l_{1}, l_{2}, \ldots, l_{n}\right] .
$$

Then it follows that the general solution of (3.8) is

$$
Y(x)=\left[\begin{array}{cc}
e^{\omega_{1} \rho x \Lambda} & 0_{n} \\
0_{n} & e^{\omega_{2} \rho x \Lambda}
\end{array}\right] \boldsymbol{C}_{1}+\left[\begin{array}{cc}
e^{\omega_{3} \rho x \Lambda} & 0_{n} \\
0_{n} & e^{\omega_{4} \rho x \Lambda}
\end{array}\right] \boldsymbol{C}_{2},
$$

where $\boldsymbol{C}_{1}$ and $\boldsymbol{C}_{2}$ are arbitrary constant $(2 n \times 1)$-vectors and

$$
e^{\omega_{j} \rho x \Lambda}=\operatorname{diag}\left\{e^{\omega_{j} \rho l_{1} x}, \ldots, e^{\omega_{j} \rho l_{n} x}\right\}, \quad j=1,2,3,4 .
$$

Hence the general solution of the governing equation of (3.5) is

$$
\begin{aligned}
{\left[\begin{array}{l}
\boldsymbol{u} \\
\boldsymbol{v}
\end{array}\right]=} & S Y(x)=\left[\begin{array}{cc}
e^{\omega_{1} \rho x \Lambda} & e^{\omega_{2} \rho x \Lambda} \\
-i P e^{\omega_{1} \rho x \Lambda} & i P e^{\omega_{2} \rho x \Lambda}
\end{array}\right] \boldsymbol{C}_{1} \\
& +\left[\begin{array}{cc}
e^{\omega_{3} \rho x \Lambda} & e^{\omega_{4} \rho x \Lambda} \\
-i P e^{\omega_{3} \rho x \Lambda} & i P e^{\omega_{4} \rho x \Lambda}
\end{array}\right] \boldsymbol{C}_{2} .
\end{aligned}
$$

In order for (3.11) to be a solution of (3.5), the constant vectors $\boldsymbol{C}_{1}$ and $\boldsymbol{C}_{2}$ should be chosen so that the boundary conditions of (3.5) be satisfied. To this end, let

$$
\begin{array}{cc}
A=\left[\begin{array}{ll}
A_{11} & A_{12} \\
A_{21} & A_{22}
\end{array}\right], & B=\left[\begin{array}{ll}
B_{11} & B_{12} \\
B_{21} & B_{22}
\end{array}\right], \\
E=\left[\begin{array}{ll}
E_{11} & E_{12} \\
E_{21} & F_{22}
\end{array}\right], & F=\left[\begin{array}{ll}
F_{11} & F_{12} \\
F_{21} & F_{22}
\end{array}\right], \\
\boldsymbol{C}_{1}=\left[\boldsymbol{c}_{1}, \boldsymbol{c}_{2}\right]^{T}, & \boldsymbol{C}_{2}=\left[\begin{array}{cc}
\boldsymbol{c}_{3}, \boldsymbol{c}_{4}
\end{array}\right]^{T},
\end{array}
$$

where $A_{k l}, B_{k l}, E_{k l}, F_{k l}, k, l=1,2$, are $(n \times n)$-matrices and

$$
\boldsymbol{c}_{j}=\left(c_{j 1}, \cdots, c_{j n}\right)^{T}, \quad j=1,2,3,4 .
$$

Then by the conditions

$$
[A, B]\left[\boldsymbol{u}_{x}(0), \boldsymbol{v}_{x}(0), \boldsymbol{u}(0), \boldsymbol{v}(0)\right]^{T}=0,
$$




$$
[E, F]\left[\boldsymbol{u}_{x}(1), \boldsymbol{v}_{x}(1), \boldsymbol{u}(1), \boldsymbol{v}(1)\right]^{T}=0,
$$

we obtain

$$
M C=0,
$$

where $\boldsymbol{C}=\left[\boldsymbol{c}_{1}, \boldsymbol{c}_{2}, \boldsymbol{c}_{3}, \boldsymbol{c}_{4}\right]^{T}$ and

$$
\begin{gathered}
M=\left[M_{1}, M_{2}, M_{3}, M_{4}\right], \\
M_{1}=\left[\begin{array}{c}
\rho \omega_{1} \Lambda\left(A_{11}-i P A_{12}\right)+B_{11}-i P B_{12} \\
\rho \omega_{1} \Lambda\left(A_{21}-i P A_{22}\right)+B_{21}-i P B_{22} \\
\left.\rho \omega_{1} \Lambda\left(E_{11}-i P E_{12}\right)+F_{11}-i P F_{12}\right] e^{\omega_{1} \rho \Lambda} \\
\left.\rho \omega_{1} \Lambda\left(E_{21}-i P E_{22}\right)+F_{21}-i P F_{22}\right] e^{\omega_{1} \rho \Lambda}
\end{array}\right], \\
M_{2}=\left[\begin{array}{c}
\rho \omega_{2} \Lambda\left(A_{11}+i P A_{12}\right)+B_{11}+i P B_{12} \\
\rho \omega_{2} \Lambda\left(A_{21}+i P A_{22}\right)+B_{21}+i P B_{32} \\
\left.\rho \omega_{2} \Lambda\left(E_{11}+i P E_{12}\right)+F_{11}+i P F_{12}\right] e^{\omega_{2} \rho \Lambda} \\
\left.\rho \omega_{2} \Lambda\left(E_{21}+i P E_{22}\right)+F_{21}+i P F_{22}\right] e^{\omega_{2} \rho \Lambda}
\end{array}\right], \\
M_{3}=\left[\begin{array}{c}
\rho \omega_{3} \Lambda\left(A_{11}-i P A_{12}\right)+B_{11}-i P B_{12} \\
\rho \omega_{3} \Lambda\left(A_{21}-i P A_{22}\right)+B_{21}-i P B_{22} \\
\left.\rho \omega_{3} \Lambda\left(E_{11}-i P E_{12}\right)+F_{11}-i P F_{12}\right] e^{\omega_{3} \rho \Lambda} \\
\left.\rho \omega_{3} \Lambda\left(E_{21}-i P E_{22}\right)+F_{21}-i P F_{22}\right] e^{\omega_{3} \rho \Lambda}
\end{array}\right], \\
M_{4}=\left[\begin{array}{c}
\rho \omega_{4} \Lambda\left(A_{11}+i P A_{12}\right)+B_{11}+i P B_{12} \\
\rho \omega_{4} \Lambda\left(A_{21}+i P A_{22}\right)+B_{21}+i P B_{22} \\
\left.\rho \omega_{4} \Lambda\left(E_{11}+i P E_{12}\right)+F_{11}+i P F_{12}\right] e^{\omega_{4} \rho \Lambda} \\
\left.\rho \omega_{4} \Lambda\left(E_{21}+i P E_{22}\right)+F_{21}+i P F_{22}\right] e^{\omega_{4} \rho \Lambda}
\end{array}\right] .
\end{gathered}
$$

It is seen that $\boldsymbol{C} \neq 0$ if and only if $\operatorname{det}(M)=0$. By (3.4),

$$
e^{\omega_{1} \rho}=O(1), \quad e^{\omega_{2} \rho}=O\left(e^{-c|\rho|}\right) \quad \text { as } \quad|\rho| \rightarrow \infty
$$

for some constant $c>0$, and, therefore,

$$
\operatorname{det}\left(e^{\left(\omega_{1}+\omega_{2}\right) \rho \Lambda}\right) \operatorname{det} M=\operatorname{det}\left[M_{1}^{\prime} M_{2}^{\prime} M_{3}^{\prime} M_{4}^{\prime}\right]+O\left(e^{-c|\rho|}\right),
$$

where

$$
\begin{gathered}
M_{1}^{\prime}=M_{1}, \quad M_{3}^{\prime}=M_{3} \cdot e^{\omega_{1} \rho \Lambda}, \\
M_{2}^{\prime}=\left[\begin{array}{c}
\rho \omega_{2} \Lambda\left(A_{11}+i P A_{12}\right)+B_{11}+i P B_{12} \\
\rho \omega_{2} \Lambda\left(A_{21}+i P A_{22}\right)+B_{21}+i P B_{22} \\
0 \\
0
\end{array}\right], \\
M_{4}^{\prime}=\left[\begin{array}{c}
0 \\
0 \\
-\rho \omega_{2} \Lambda\left(E_{11}+i P E_{12}\right)+F_{11}+i P F_{12} \\
-\rho \omega_{2} \Lambda\left(E_{21}+i P E_{22}\right)+F_{21}+i P F_{22}
\end{array}\right] .
\end{gathered}
$$


Now setting $e^{2 \omega_{1} \rho}=y$ and taking the dominant terms of $\rho$, we find that $\operatorname{det}\left(e^{\left(\omega_{1}+\omega_{2}\right) \rho \Lambda}\right) \operatorname{det} M=0$ can be written equivalently as (3.2) for some $f$ claimed. The result follows.

The method adopted in proving Proposition 1 is from [13], which much simplifies the analysis in [20]. Our next task is to estimate asymptotically the distribution of eigenvalues $\mathcal{A}$. In general, this seems to be impossible. In the next section, we show by an example that the general collinear EulerBernoulli beam equation with linear joints that was studied in [20] can be put into the form of system (1.2). The main result of [20] says that if all $l_{j}, j=1,2, \ldots, n$, are the same, then there is at most $n$ streams of eigenfrequencies, each lying asymptotically on a vertical line. More generally, if $l_{1}: l_{2}: \cdots: l_{n}=p_{1}: p_{2}: \cdots: p_{n}$, where $p_{i}$ are all integers, then there is at most $p_{1}+p_{2}+\cdots+p_{n}$ streams of vertical eigenfrequencies. However, if the ratios of $l_{j}$ are irrational, then no distinct streams occur, and the eigenfrequencies form a chaotic pattern. In particular, if all $l_{j}, j=1,2, \ldots, n$, are integers, then $f(y)$ is a polynomial of degree less than $\sum_{i=1}^{n} l_{i}=L_{n}$ and there are at most $L_{n}$ "streams" of vertical eigenfrequencies. This result is deduced by a key assumption that $f(y)$ has only simple roots for all $\rho$ with sufficiently large moduli.

\section{Application to a system of $N$-Connected Beams}

In this section, we apply the results of the previous sections to a system of serially connected beams with linear feedback control at the joint points discussed in [20] as its type II of four types of general collinear EulerBernoulli beam equation with linear joints. That is, at each joint point, it is assumed that both displacement and bending moment are continuous, but rotation and shear force are discontinuous. We find not only the asymptotic expansion of eigenvalues which is the major concern of [20], but also the asymptotic expansion of the corresponding eigenfunctions. The governing equation reads

$$
y_{t t}(x, t)+y_{x x x x}(x, t)=0, \quad L_{j-1}<x<L_{j}, \quad j=1,2, \ldots, n .
$$

The boundary conditions are

$$
\left\{\begin{array}{l}
y(0)=y_{x x}(0)=0, \\
y_{x}\left(L_{n}\right)=y_{x x x}\left(L_{n}\right)=0 .
\end{array}\right.
$$


The linear feedback controls at the joint points $L_{j}, j=1, \ldots, n-1$, take the form

$$
\left\{\begin{array}{l}
y\left(L_{j}^{-}, t\right)=y\left(L_{j}^{+}, t\right), \\
y_{x x}\left(L_{j}^{-}, t\right)=y_{x x}\left(L_{j}^{+}, t\right), \\
y_{t x}\left(L_{j}^{+}, t\right)-y_{t x}\left(L_{j}^{-}, t\right)=(-1)^{j} r_{j} y_{t}\left(L_{j}^{-}, t\right)+p_{j}^{2} y_{x x}\left(L_{j}^{-}, t\right), \\
y_{x x x}\left(L_{j}^{+}, t\right)-y_{x x x}\left(L_{j}^{-}, t\right)=-q_{j}^{2} y_{t}\left(L_{j}^{-}, t\right)+(-1)^{j} s_{j} y_{x x}\left(L_{j}^{-}, t\right),
\end{array}\right.
$$

where $0=L_{0}<L_{1}<\cdots<L_{n}$ and

$$
\begin{gathered}
p_{j}^{2} \geq 0, \quad q_{j}^{2} \geq 0, \quad p_{j}^{2}+q_{j}^{2}>0, \quad r_{j}, s_{j} \in \mathbb{R}, \\
p_{j}^{2} \alpha^{2}+q_{j}^{2} \beta^{2}+\left(r_{j}-s_{j}\right) \alpha \beta \geq 0 \quad \forall \alpha, \beta \in \mathbb{R} .
\end{gathered}
$$

Let us define the energy of system (4.1)-(4.4) as

$$
E(t)=\frac{1}{2} \sum_{j=1}^{n} \int_{L_{j-1}}^{L_{j}}\left[y_{t}^{2}(x, t)+y_{x x}^{2}(x, t)\right] d x .
$$

Then a simple computation shows that $\dot{E}(t) \leq 0$ and hence the system is dissipative.

Without loss of generality, we may assume that $n$ is odd. For $j=1,2, \ldots, n$, we set

$$
\left\{\begin{aligned}
u_{j}(x, t) & =\frac{1}{2}\left[y_{t}\left(L_{j}+\frac{(-1)^{j}-1}{2} l_{j}+(-1)^{j+1} l_{j} x, t\right)\right. \\
& \left.+\frac{(-1)^{j+1}}{l_{j}^{2}} y_{x x}\left(L_{j}+\frac{(-1)^{j}-1}{2} l_{j}+(-1)^{j+1} l_{j} x, t\right)\right], \\
v_{j}(x, t) & =\frac{1}{2}\left[y_{t}\left(L_{j}+\frac{(-1)^{j}-1}{2} l_{j}+(-1)^{j+1} l_{j} x, t\right)\right. \\
& \left.-\frac{(-1)^{j+1}}{l_{j}^{2}} y_{x x}\left(L_{j}+\frac{(-1)^{j}-1}{2} l_{j}+(-1)^{j+1} l_{j} x, t\right)\right],
\end{aligned}\right.
$$

where $l_{j}=L_{j}-L_{j-1}, j=1,2, \ldots, n, 0 \leq x \leq 1$. Then system (4.1)-(4.4) can be transformed into the form of $(1.2)$ with the following $(2 n \times 2 n)$ matrices:

$$
A=\left[\begin{array}{cccccccccc}
0 & 0 & 0 & \ldots & 0 & 0 & 0 & 0 & \ldots & 0 \\
0 & P_{21} & 0 & \ldots & 0 & 0 & P_{22} & 0 & \ldots & 0 \\
0 & 0 & P_{41} & \ldots & 0 & 0 & 0 & P_{42} & \ldots & 0 \\
\vdots & \vdots & \vdots & \ddots & \vdots & \vdots & \vdots & \vdots & \ddots & \vdots \\
0 & 0 & 0 & \ldots & P_{(n-1) 1} & 0 & 0 & 0 & \ldots & P_{(n-1) 2}
\end{array}\right]
$$




$$
\begin{aligned}
& B=\left[\begin{array}{cccccccccc}
P_{n 1} & 0 & 0 & \ldots & 0 & P_{n 2} & 0 & 0 & \ldots & 0 \\
0 & \tilde{P}_{21} & 0 & \ldots & 0 & 0 & \tilde{P}_{22} & 0 & \ldots & 0 \\
0 & 0 & \tilde{P}_{41} & \ldots & 0 & 0 & 0 & \tilde{P}_{42} & \ldots & 0 \\
\vdots & \vdots & \vdots & \ddots & \vdots & \vdots & \vdots & \vdots & \ddots & \vdots \\
0 & 0 & 0 & \ldots & \tilde{P}_{(n-1) 1} & 0 & 0 & 0 & \ldots & \tilde{P}_{(n-1) 2}
\end{array}\right] \\
& E=\left[\begin{array}{cccccccccc}
P_{11} & 0 & \ldots & 0 & 0 & P_{12} & 0 & \ldots & 0 & 0 \\
0 & P_{31} & \ldots & 0 & 0 & 0 & P_{32} & \ldots & 0 & 0 \\
\vdots & \vdots & \ddots & \vdots & \vdots & \vdots & \vdots & \ddots & \vdots & \vdots \\
0 & 0 & \ldots & P_{(n-2) 1} & 0 & 0 & 0 & \ldots & P_{(n-2) 2} & 0 \\
0 & 0 & \ldots & 0 & P_{n 1} & 0 & 0 & \ldots & 0 & P_{n 2}
\end{array}\right] \\
& F=\left[\begin{array}{cccccccccc}
\tilde{P}_{11} & 0 & \ldots & 0 & 0 & \tilde{P}_{12} & 0 & \ldots & 0 & 0 \\
0 & \tilde{P}_{31} & \ldots & 0 & 0 & 0 & \tilde{P}_{32} & \ldots & 0 & 0 \\
\vdots & \vdots & \ddots & \vdots & \vdots & \vdots & \vdots & \ddots & \vdots & \vdots \\
0 & 0 & \ldots & \tilde{P}_{(n-2) 1} & 0 & 0 & 0 & \ldots & \tilde{P}_{(n-2) 2} & 0 \\
0 & 0 & \ldots & 0 & 0 & 0 & 0 & \ldots & 0 & 0
\end{array}\right],
\end{aligned}
$$

where for $j=1,2, \ldots, n-1$,

$$
\begin{gathered}
P_{n 1}=\left[\begin{array}{l}
1 \\
1
\end{array}\right], \quad P_{n 2}=\left[\begin{array}{c}
1 \\
-1
\end{array}\right], \\
P_{j 1}=\left[\begin{array}{cc}
0 & 0 \\
0 & 0 \\
\frac{1}{l_{j}} & \frac{1}{l_{j+1}} \\
\frac{-1}{l_{j}} & \frac{1}{l_{j+1}}
\end{array}\right], \quad P_{j 2}=\left[\begin{array}{cc}
0 & 0 \\
0 & 0 \\
\frac{1}{l_{j}} & \frac{1}{l_{j+1}} \\
\frac{1}{l_{j}} & \frac{-1}{l_{j+1}}
\end{array}\right], \\
\tilde{P}_{j 1}=\left[\begin{array}{cc}
1 & -1 \\
1 & 1 \\
p_{j}^{2}-r_{j} & 0 \\
q_{j}^{2}+s_{j} & 0
\end{array}\right], \quad \tilde{P}_{j 2}=\left[\begin{array}{cc}
1 & -1 \\
-1 & -1 \\
-p_{j}^{2}-r_{j} & 0 \\
q_{j}^{2}-s_{j} & 0
\end{array}\right] .
\end{gathered}
$$

In the rest of this paper, we are limit ourselves to system (4.1)-(4.4) but keep the notation of system (1.2) with $A, B, E, F$ specified by (4.6). Divide by $\rho \omega_{1}$ both sides of those equations which contain nonzero factors $\rho$ in the system $\tilde{M} \boldsymbol{C}=0$; then (3.12) becomes

$$
\tilde{M} \boldsymbol{C}=0,
$$

where

$$
\tilde{M}=\left[\begin{array}{llll}
M_{1} & M_{2} & M_{3} & M_{4}
\end{array}\right],
$$

and for $1 \leq k \leq 4$, 


$$
\begin{aligned}
& M_{k}=\left[\begin{array}{cccc}
Q_{0 k} & 0 & 0 & \cdots \\
0 & Q_{2 k} & R_{2 k} & \cdots \\
\vdots & \vdots & \vdots & \ddots \\
0 & 0 & 0 & \cdots \\
Q_{1 k} e^{\omega_{k} \rho l_{1}} & R_{1 k} e^{\omega_{k} \rho l_{2}} & 0 & \cdots \\
\vdots & \vdots & \vdots & \ddots \\
0 & 0 & 0 & \cdots \\
0 & 0 & 0 & \cdots
\end{array}\right. \\
& \left.\begin{array}{ccc}
0 & 0 & 0 \\
0 & 0 & 0 \\
\vdots & \vdots & \vdots \\
0 & Q_{(n-1) k} & R_{(n-1) k} \\
0 & 0 & 0 \\
\vdots & \vdots & \vdots \\
Q_{(n-2) k} e^{\omega_{k} \rho l_{n-2}} & R_{(n-2) k} e^{\omega_{k} \rho l_{n-1}} & 0 \\
0 & 0 & Q_{n 1} e^{\omega_{k} \rho l_{n}}
\end{array}\right]_{4 n \times n}
\end{aligned}
$$

with

$$
\begin{gathered}
Q_{01}=\left[\begin{array}{ll}
1-i & 1+i
\end{array}\right]^{T}, \quad Q_{02}=\left[\begin{array}{cc}
1+i & 1-i^{T}
\end{array}\right]^{T}, \\
Q_{03}=Q_{01}, \quad Q_{04}=Q_{02}, \quad Q_{n 1}=Q_{01}, \\
Q_{n 2}=Q_{02} \cdot i, \quad Q_{n 3}=-Q_{n 1}, \quad Q_{n 4}=-Q_{n 2} .
\end{gathered}
$$

For $j=1,3, \ldots, n-2, l=2,4, \ldots, n-1$,

$$
\begin{aligned}
& Q_{j 1}=\left[1-i+\frac{(1+i) p_{j}^{2}-(1-i) r_{j}}{\rho \omega_{1}},\right. \\
& \left.-(1+i)+\frac{(1-i) q_{j}^{2}+(1+i) s_{j}}{\rho \omega_{1}}, \quad 1-i, \quad 1+i\right]^{T}, \\
& Q_{j 2}=\left[-(1-i)+\frac{(1-i) p_{j}^{2}-(1+i) r_{j}}{\rho \omega_{1}},\right. \\
& \left.-(1+i)+\frac{(1+i) q_{j}^{2}+(1-i) s_{j}}{\rho \omega_{1}}, \quad 1+i, \quad 1-i\right]^{T}, \\
& Q_{j 3}=\left[-(1-i)+\frac{(1+i) p_{j}^{2}-(1-i) r_{j}}{\rho \omega_{1}},\right. \\
& \left.1+i+\frac{(1-i) q_{j}^{2}+(1+i) s_{j}}{\rho \omega_{1}}, \quad 1-i, \quad 1+i\right]^{T}, \\
& Q_{j 4}=\left[1-i+\frac{(1-i) p_{j}^{2}-(1+i) r_{j}}{\rho \omega_{1}},\right.
\end{aligned}
$$




$$
\begin{aligned}
& \left.1+i+\frac{(1+i) q_{j}^{2}+(1-i) s_{j}}{\rho \omega_{1}}, \quad 1+i, \quad 1-i\right]^{T} \\
& Q_{l 1}=\left[1+i+\frac{(1-i) p_{l}^{2}-(1+i) r_{l}}{\rho \omega_{1}}\right. \\
& \left.-(1-i)+\frac{(1+i) q_{l}^{2}+(1-i) s_{l}}{\rho \omega_{1}}, \quad 1+i, \quad 1-i\right]^{T}, \\
& Q_{l 2}=\left[1+i+\frac{(1+i) p_{l}^{2}-(1-i) r_{l}}{\rho \omega_{1}}\right. \\
& \left.1-i+\frac{(1-i) q_{l}^{2}+(1+i) s_{l}}{\rho \omega_{1}}, \quad 1-i, \quad 1+i\right]^{T} \\
& Q_{l 3}=\left[-(1+i)+\frac{(1-i) p_{l}^{2}-(1+i) r_{l}}{\rho \omega_{1}},\right. \\
& \left.1-i+\frac{(1+i) q_{l}^{2}+(1-i) s_{l}}{\rho \omega_{1}}, \quad 1+i, \quad 1-i\right]^{T}, \\
& Q_{l 4}=\left[-(1+i)+\frac{(1+i) p_{l}^{2}-(1-i) r_{l}}{\rho \omega_{1}},\right. \\
& \left.-(1-i)+\frac{(1-i) q_{l}^{2}+(1+i) s_{l}}{\rho \omega_{1}}, \quad 1-i, \quad 1+i\right]^{T} \text {; }
\end{aligned}
$$

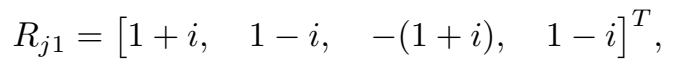

$$
\begin{aligned}
& R_{j 2}=\left[\begin{array}{lll}
1+i, & -(1-i), \quad-(1-i), \quad 1+i
\end{array}\right]^{T},
\end{aligned}
$$

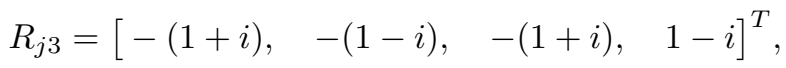

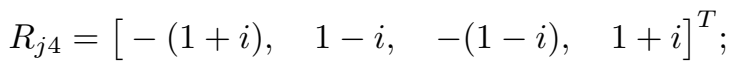

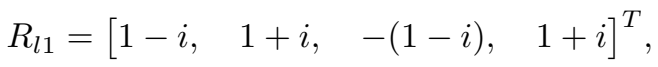

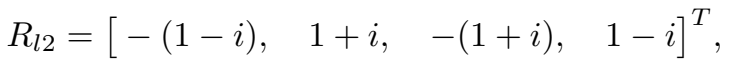

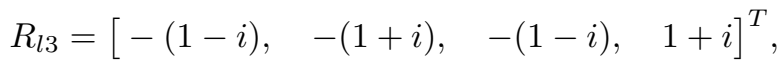

$$
\begin{aligned}
& R_{l 4}=\left[\begin{array}{lll}
1-i, & -(1+i), \quad-(1+i), & 1-i
\end{array}\right]^{T} \text {. }
\end{aligned}
$$

Lemma 1. Let $\mathcal{A}$ be the operator associated with system (4.1)-(4.4) and $\lambda=\rho^{2} \in \sigma(\mathcal{A}), \pi / 4 \leq \arg (\rho) \leq \pi / 2$. Then

$$
\rho_{k}=\frac{(k+1 / 2) \pi \omega_{1}}{L_{n}}+O\left(k^{-1}\right),
$$

where $k$ are sufficiently large positive integers. 
Proof. Note that $\lambda=\rho^{2} \in \sigma(\mathcal{A})$ if and only if $\operatorname{det} \tilde{M}=0$. Now multiplying $\operatorname{det} \tilde{M}$ by $e^{\left(\omega_{1}+\omega_{2}\right) \rho L_{n}}$, we can obtain (3.15) with $M_{j}^{\prime}, j=1,2,3,4$, defined by

$$
M_{1}^{\prime}=\tilde{M}_{1}, \quad M_{2}^{\prime}=\tilde{M}_{2}, \quad M_{3}^{\prime}=\tilde{M}_{3} \cdot e^{\rho \omega_{1} \Lambda}, \quad M_{4}^{\prime}=\tilde{M}_{4} \cdot e^{\rho \omega_{2} \Lambda},
$$

where $\tilde{M}_{j}, j=1,2,3,4$, are obtained from corresponding $M_{j}$ in (4.9) after setting $Q_{j 2}=R_{j 2}=0$ for odd $j$ and $Q_{j 4}=R_{j 4}=0$ for even $j$. The others remain the same as in (4.10)-(4.14).

After a straightforward computation, we obtain

$$
\operatorname{det}\left[M_{1}^{\prime}, M_{2}^{\prime}, M_{3}^{\prime}, M_{4}^{\prime}\right]=\operatorname{det} M^{\prime}+O\left(\rho^{-1}\right)
$$

with the following $(4 n \times 4 n)$-matrix:

$$
M^{\prime}=\left[\begin{array}{ccccccc}
N_{0} & 0 & 0 & \ldots & 0 & 0 & 0 \\
N_{11} & N_{12} & 0 & \ldots & 0 & 0 & 0 \\
0 & N_{21} & N_{22} & \ldots & 0 & 0 & 0 \\
\vdots & \vdots & \vdots & \ddots & \vdots & \vdots & \vdots \\
0 & 0 & 0 & \ldots & N_{(n-2) 1} & N_{(n-2) 2} & 0 \\
0 & 0 & 0 & \ldots & 0 & N_{(n-1) 1} & N_{(n-1) 2} \\
0 & 0 & 0 & \ldots & 0 & 0 & N_{n}
\end{array}\right],
$$

where

$$
\begin{aligned}
N_{0}= & {\left[Q_{01}, Q_{02}, Q_{03} \cdot e^{\omega_{1} \rho l_{1}}, 0\right], } \\
N_{n}= & {\left[Q_{n 1} \cdot e^{\omega_{1} \rho l_{n}}, 0, Q_{n 3}, Q_{n 4}\right], } \\
N_{j 1}= & {\left[Q_{j 1}^{\prime} \cdot e^{\omega_{1} \rho l_{j}}, 0, Q_{j 3}^{\prime}, Q_{j 4}^{\prime}\right], } \\
N_{j 2}= & {\left[R_{j 1} \cdot e^{\omega_{1} \rho l_{j+1}}, 0, R_{j 3}, R_{j 4}\right], } \\
N_{l 1}= & {\left[Q_{l 1}^{\prime}, Q_{l 2}^{\prime}, Q_{l 3}^{\prime} \cdot e^{\omega_{1} \rho l_{l}}, 0\right], } \\
N_{l 2}= & {\left[R_{l 1}, R_{l 2}, R_{l 3} \cdot e^{\omega_{1} \rho l_{l+1}}, 0\right], } \\
& \quad j=1,3, \ldots, n-2, \quad l=2,4, \ldots, n-1,
\end{aligned}
$$

and $Q_{0 l}, Q_{n l}$, and $R_{j l}$ are the same as in (4.10), (4.13), and (4.14), and $Q_{j l}^{\prime}$ are given by

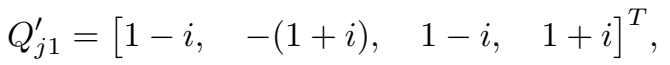

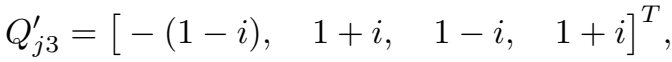

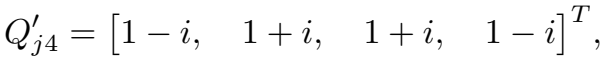

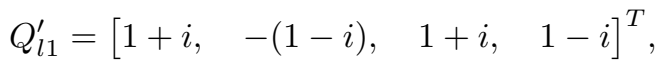

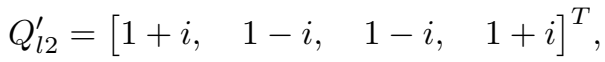

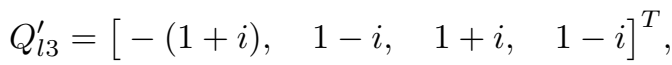

$$
\begin{aligned}
& j=1,3, \ldots, n-2, \quad l=2,4, \ldots, n-1 .
\end{aligned}
$$


A similar computation as in [20] shows that

$$
\operatorname{det} M^{\prime}=(-1)^{(n+1) / 2} \cdot 16^{n} \cdot i \cdot \operatorname{det} S,
$$

where $S$ is a $(2 n \times 2 n)$-matrix of the form (4.18) with $N_{j}$ being replaced by $N_{j}^{\prime}$ :

$$
\begin{gathered}
N_{0}^{\prime}=\left[\begin{array}{ll}
1, & e^{\omega_{1} \rho l_{1}}
\end{array}\right], \quad N_{n}^{\prime}=\left[\begin{array}{ll}
-e^{\omega_{1} \rho l_{n}}, & 1
\end{array}\right], \\
N_{k 1}^{\prime}=\left[\begin{array}{cc}
-(1+i) e^{\omega_{1} \rho l_{k}} & 1+i \\
(1+i) e^{\omega_{1} \rho l_{k}} & 1+i
\end{array}\right], \quad N_{k 2}^{\prime}=\left[\begin{array}{cc}
(1-i) e^{\omega_{1} \rho l_{k+1}} & -(1-i) \\
(1-i) e^{\omega_{1} \rho l_{k+1}} & 1-i
\end{array}\right], \\
N_{l 1}^{\prime}=\left[\begin{array}{cc}
-1+i & (1-i) e^{\omega_{1} \rho l_{l}} \\
1-i & (1-i) e^{\omega_{1} \rho l_{l}}
\end{array}\right], \quad N_{l 2}^{\prime}=\left[\begin{array}{cc}
1+i & -(1+i) e^{\omega_{1} \rho l_{l+1}} \\
1+i & (1+i) e^{\omega_{1} \rho l_{l+1}}
\end{array}\right], \\
k=1,3, \ldots, n-2, \quad l=2,4, \ldots, n-1 .
\end{gathered}
$$

Using the same approach as in [20], we can obtain

$$
\begin{aligned}
\operatorname{det} S & =4^{n-1} \cdot S_{0} \cdot S_{1} \cdots S_{n} \\
& =4^{n-1} \cdot\left(e^{2 \omega_{1} \rho\left(l_{1}+\cdots+l_{n}\right)}+1\right)=4^{n-1} \cdot\left(e^{2 \omega_{1} \rho L_{n}}+1\right),
\end{aligned}
$$

where

$$
\begin{gathered}
S_{0}=\left[\begin{array}{ll}
1, & e^{\omega_{1} \rho l_{1}}
\end{array}\right], \quad S_{n}=\left[\begin{array}{ll}
1, & e^{\omega_{1} \rho l_{n}}
\end{array}\right]^{T}, \\
S_{j}=\left[\begin{array}{cc}
0 & 1 \\
e^{\omega_{1} \rho\left(l_{j}+l_{j+1}\right)} & 0
\end{array}\right], \quad j=1,3, \ldots, n-2, \\
S_{l}=\left[\begin{array}{cc}
0 & e^{\omega_{1} \rho\left(l_{j}+l_{j+1}\right)} \\
1 & 0
\end{array}\right], \quad l=2,4, \ldots, n-1 .
\end{gathered}
$$

Combining (4.17), (4.19), and (4.20), we finally find that $\operatorname{det} \tilde{M}=0$ is equivalent to

$$
e^{2 \omega_{1} \rho L_{n}}+1+O\left(\rho^{-1}\right)=0 .
$$

Therefore, in this case $f(y)=y^{L_{n}}+1$ and $f(y)=0$ does possess only simple zeros. By the Rouché theorem, the solution of (4.22) can be found as

$$
2 \omega_{1} \rho L_{n}=(2 k+1) \pi i+O\left(\rho^{-1}\right)
$$

for sufficiently large positive integers $k$, which proves Lemma 1 .

Now we are in a position to estimate asymptotically the eigenfunctions $\left[\boldsymbol{u}_{k}, \boldsymbol{v}_{k}\right]^{T}$ corresponding to $\lambda_{k}=\rho_{k}^{2}$ with $\rho_{k}$ given by (4.15).

Lemma 2. Let $\left[\boldsymbol{u}_{k}, \boldsymbol{v}_{k}\right]^{T}$ be the eigenfunctions corresponding to $\lambda_{k}=\rho_{k}^{2}$ with $\rho_{k}$ given by (4.15) for all sufficiently large positive integers $k$ :

$$
\boldsymbol{u}_{k}=\left[u_{1 k}, \ldots, u_{n k}\right]^{T}, \quad \boldsymbol{v}_{k}=\left[v_{1 k}, \ldots, v_{n k}\right]^{T} .
$$

Then for odd $j$, we have

$$
u_{j k}(x)=\sin \frac{(k+1 / 2) \pi L_{j}}{L_{n}}\left\{\sin \frac{(k+1 / 2) \pi\left(L_{j-1}+l_{j} x\right)}{L_{n}}\right.
$$




$$
\begin{aligned}
& \left.+i e^{-\frac{(k+1 / 2) \pi l_{j} x}{L_{n}}} \cdot \sin \frac{(k+1 / 2) \pi L_{j-1}}{L_{n}}\right\}+O\left(k^{-1}\right), \\
v_{j k}(x) & =-i \cdot \sin \frac{(k+1 / 2) \pi L_{j}}{L_{n}}\left\{\sin \frac{(k+1 / 2) \pi\left(L_{j-1}+l_{j} x\right)}{L_{n}}\right. \\
& \left.-i e^{-\frac{(k+1 / 2) \pi l_{j} x}{L_{n}}} \cdot \sin \frac{(k+1 / 2) \pi L_{j-1}}{L_{n}}\right\}+O\left(k^{-1}\right) ;
\end{aligned}
$$

for even $j$, we have

$$
\begin{aligned}
u_{j k}(x) & =\sin \frac{(k+1 / 2) \pi L_{j}}{L_{n}}\left\{\sin \frac{(k+1 / 2) \pi\left(L_{j}-l_{j} x\right)}{L_{n}}\right. \\
& \left.-i e^{-\frac{(k+1 / 2) \pi l_{j}(1-x)}{L_{n}}} \cdot \sin \frac{(k+1 / 2) \pi L_{j-1}}{L_{n}}\right\}+O\left(k^{-1}\right), \\
v_{j k}(x) & =i \cdot \sin \frac{(k+1 / 2) \pi L_{j}}{L_{n}}\left\{\sin \frac{(k+1 / 2) \pi\left(L_{j}-l_{j} x\right)}{L_{n}}\right. \\
& \left.+i e^{-\frac{(k+1 / 2) \pi l_{j}(1-x)}{L_{n}}} \cdot \sin \frac{(k+1 / 2) \pi L_{j-1}}{L_{n}}\right\}+O\left(k^{-1}\right),
\end{aligned}
$$

where $k \in \mathbb{Z}$.

Proof. Since some calculations in proving Lemma 2 and Lemma 1 are overwhelming, we postpone the proof of Lemma 2 in the Appendix.

We summarize these results as the following Theorem 2 .

Theorem 2. Let $\mathcal{A}$ be the operator of system (4.1)-(4.4), $\sigma(\mathcal{A})=$ $\left\{\lambda_{k}, \overline{\lambda_{k}}\right\}$. Let $\left\{\left[\boldsymbol{u}_{k}, \boldsymbol{v}_{k}\right]^{T},\left[\overline{\boldsymbol{u}}_{k}, \overline{\boldsymbol{v}}_{k}\right]^{T}\right\}$ be the corresponding eigenfunctions. Then $\lambda_{k}=\rho_{k}^{2}$ and $\boldsymbol{u}_{k}=\left[u_{1 k}, \ldots, u_{n k}\right]^{T}$ have the asymptotic expansions (4.15) and (4.23), respectively, for sufficiently large positive integers $k$.

\section{Riesz Basis GENERATION FOR System (4.1)-(4.4)}

Let us recall that for a closed linear operator $A$ in a Hilbert space $\boldsymbol{H}$, a nonzero $x \in \boldsymbol{H}$ is called a generalized eigenvector of $A$, corresponding to an eigenvalue $\lambda$ of $A$ with finite algebraic multiplicity, if there is a positive integer $n$ such that $(\lambda-A)^{n} x=0$. A sequence $\left\{\phi_{n}\right\}_{1}^{\infty}$ in $\boldsymbol{H}$ is called a Riesz basis for $\boldsymbol{H}$ if there exists an orthonormal basis $\left\{e_{n}\right\}_{1}^{\infty}$ in $\boldsymbol{H}$ and a linear bounded invertible operator in $\boldsymbol{H}$ such that

$$
T \phi_{n}=e_{n}, \quad n=1,2, \ldots
$$

For a linear operator $A$ in a Hilbert space $\boldsymbol{H}$, let $\left\{\lambda_{n}\right\}_{n=1}^{\infty}=\sigma(A)$ with $\lambda_{n} \neq$ $\lambda_{m}$ for $n \neq m$ be the spectrum of $A$. Suppose that the algebraic multiplicity of $\lambda_{n}$ is $m_{n}(<\infty)$. Let $\left\{\psi_{n_{i}}\right\}_{1}^{m_{n}}$ be the set of generalized eigenvectors of $A$ associated with $\lambda_{n}$. Then if $\left\{\psi_{n_{i}} \mid 1 \leq i \leq m_{n}, n=1,2, \ldots\right\}$ form a Riesz 
basis for $\boldsymbol{H}$, then the $C_{0}$-semigroup $e^{A t}$ generated by $A$ can be represented as

$$
e^{A t} x=\sum_{n=1}^{\infty} e^{\lambda_{n} t} \sum_{j=1}^{m_{n}} a_{n j} f_{n j}(t) \psi_{n j} \quad \forall x=\sum_{n=1}^{\infty} \sum_{j=1}^{m_{n}} a_{n j} \psi_{n j} \in \boldsymbol{H},
$$

where $f_{n j}(t)$ are polynomials of order not greater than $m_{n}$. In particular, if $m_{n}=1$ for all sufficiently large $n$, then the the spectrum-determined growth condition holds, i.e. $\omega(A)=S(A)$, where $\omega(A)$ is the growth bound of $e^{A t}, S(A)$ is the spectral bound of $A$.

The following result developed recently in [7] turns out to be very useful for the verification of Riesz basis generation for beam equations.

Theorem 3. Let $A$ be a densely defined discrete operator. If there are an integer $N \geq 0$ and a sequence of generalized eigenvectors $\left\{\psi_{n}\right\}_{n=N+1}^{\infty}$ of A such that

$$
\sum_{N+1}^{\infty}\left\|\phi_{n}-\psi_{n}\right\|^{2}<\infty
$$

where $\left\{\phi_{n}\right\}_{n=1}^{\infty}$ is a Riesz basis for $\boldsymbol{H}$, then the following assertions hold.

(i) There are constant $M>N$ and generalized eigenvectors $\left\{\psi_{n 0}\right\}_{1}^{M}$ of A such that $\left\{\psi_{n 0}\right\}_{1}^{M} \cup\left\{\psi_{n}\right\}_{M+1}^{\infty}$ form a Riesz basis for $\boldsymbol{H}$.

(ii) Let $\left\{\psi_{n 0}\right\}_{1}^{M} \cup\left\{\psi_{n}\right\}_{M+1}^{\infty}$ correspond to the eigenvalues $\left\{\sigma_{n}\right\}_{1}^{\infty}$ of $A$. Then $\sigma(A)=\left\{\sigma_{n}\right\}_{1}^{\infty}$, where $\sigma_{n}$ is counted according to its algebraic multiplicity.

(iii) If there is an integer $M_{0}>0$ such that $\sigma_{n} \neq \sigma_{m}$ for all $m, n>M_{0}$, then there is an integer $N_{0}>M_{0}$ such that all $\sigma_{n}$ are algebraically simple for all $n>M_{0}$.

The main result of this section is the following Theorem 4 .

Theorem 4. For system (4.1)-(4.4), if there exists $j, j \in\{1, \ldots, n-1\}$, such that $\frac{L_{j}}{L_{n}}=\frac{\bar{m}}{\bar{n}}$, where $\bar{m}$ and $\bar{n}$ are coprime integers and $\bar{m}$ is odd, then the following assertions hold.

(i) There is a sequence of generalized eigenvectors of the system operator $\mathcal{A}$, which forms a Riesz basis for $\mathcal{H}$.

(ii) Let $\sigma(\mathcal{A})=\left\{\lambda_{k}, \overline{\lambda_{k}}\right\}$. Then $\lambda_{k}=\rho_{k}^{2}$ with $\rho_{k}$ given by (4.15) are algebraically simple for all sufficiently large positive integers $k$.

Therefore, for the $C_{0}$-semigroup $e^{\mathcal{A} t}$ generated by $\mathcal{A}$, the spectrumdetermined growth condition $\omega(\mathcal{A})=S(\mathcal{A})$ holds.

Proof. We show that $\left[\boldsymbol{u}_{k}, \boldsymbol{v}_{k}\right]^{T}$ determined by Theorem 2 satisfy the condition of Theorem 3 for a properly chosen reference Riesz basis. To do this, 
we define another operator $\widetilde{\mathcal{A}}: D(\widetilde{\mathcal{A}})(\subset \mathcal{H}) \rightarrow \mathcal{H}$ by the rule

$$
\widetilde{\mathcal{A}}\left[\begin{array}{l}
\boldsymbol{u} \\
\boldsymbol{v}
\end{array}\right]=K \frac{\partial^{2}}{\partial x^{2}}\left[\begin{array}{l}
\boldsymbol{u} \\
\boldsymbol{v}
\end{array}\right]
$$

where

$D(\widetilde{\mathcal{A}})=\left\{\begin{array}{l|l}{[\boldsymbol{u}, \boldsymbol{v}]^{T} \in\left(H^{2}(0,1)\right)^{2 n}} & \begin{array}{l}{[A, \widetilde{B}]\left[\boldsymbol{u}_{x}(0), \boldsymbol{v}_{x}(0), \boldsymbol{u}(0), \boldsymbol{v}(0)\right]^{T}=0,} \\ {[E, \widetilde{F}]\left[\boldsymbol{u}_{x}(1), \boldsymbol{v}_{x}(1), \boldsymbol{u}(1), \boldsymbol{v}(1)\right]^{T}=0}\end{array}\end{array}\right\}$,

where $A$ and $E$ are given in (4.6), $\widetilde{B}$ and $\widetilde{F}$ are the same as $B$ and $F$ but with $p_{j}^{2}, q_{j}^{2}, r_{j}, s_{j}, j=1, \ldots, n-1$, vanishing. Then it is easy to see that $\widetilde{\mathcal{A}}$ is skew-adjoint in $\mathcal{H}$ with compact resolvent. The results of the previous sections are still valid for the eigenvector $\left[\widetilde{\boldsymbol{u}}_{k}, \widetilde{\boldsymbol{v}}_{k}\right]^{T}$ of $\widetilde{A}$, where

$$
\widetilde{\boldsymbol{u}}_{k}=\left[\widetilde{u}_{1 k}, \ldots, \widetilde{u}_{n k}\right]^{T}, \quad \widetilde{\boldsymbol{v}}_{k}=\left[\widetilde{v}_{1 k}, \ldots, \widetilde{v}_{n k}\right]^{T}, \quad \widetilde{u}_{j k}, \widetilde{v}_{j k}, \quad j=1, \ldots, n,
$$

and without loss of generality, we assume that $k \in \mathbb{Z}$. Moreover, $\widetilde{u}_{j k}$ and $\widetilde{v}_{j k}$ have the same asymptotics as $u_{j k}$ and $v_{j k}$ given in (4.23)-(4.26).

Since there exists $j, j \in\{1, \ldots, n-1\}$, such that

$$
\frac{L_{j}}{L_{n}}=\frac{\bar{m}}{\bar{n}}, \quad \bar{m} \text { and } \bar{n} \text { are coprime integers, } \bar{m} \text { is odd, }
$$

it follows from [21] that there exists a constant $c>0$ such that

$$
\left|\sin \frac{(k+1 / 2) \pi L_{j}}{L_{n}}\right|>c \text { for all } k \in \mathbb{Z} .
$$

A simple calculation shows that

$$
\begin{aligned}
\left\|\left[\widetilde{\boldsymbol{u}}_{k}, \widetilde{\boldsymbol{v}}_{k}\right]^{T}\right\|_{\mathcal{H}}^{2} & =\sum_{j=1}^{n} \int_{0}^{1}\left(\left|\widetilde{u}_{j k}(x)\right|^{2}+\left|\widetilde{v}_{j k}(x)\right|^{2}\right) d x \\
& =\sum_{j=1}^{n}\left|\sin \frac{(k+1 / 2) \pi L_{j}}{L_{n}}\right|^{2} \cdot\left[1+O\left(k^{-1}\right)\right]
\end{aligned}
$$

as $k \rightarrow \infty$. By (5.3), we see that there exist positive constants $m$ and $M$ independent of $k$ such that

$$
m<\left\|\left[\widetilde{\boldsymbol{u}}_{k}, \widetilde{\boldsymbol{v}}_{k}\right]^{T}\right\|_{\mathcal{H}}^{2}<M \text { for all } k \in \mathbb{Z} .
$$

Since $\widetilde{\mathcal{A}}$ is skew-adjoint with compact resolvent, it follows from general operator theory that $\left[\widetilde{\boldsymbol{u}}_{k}, \widetilde{\boldsymbol{v}}_{k}\right]^{T}, k \in \mathbb{Z}$, together with their conjugates, form a Riesz basis for $\mathcal{H}$.

Furthermore, since $\left[\widetilde{\boldsymbol{u}}_{k}, \widetilde{\boldsymbol{v}}_{k}\right]^{T}$ have the same asymptotic expansions as $\left[\boldsymbol{u}_{k}, \boldsymbol{v}_{k}\right]^{T}$, we see that there exists a $N>0$ such that

$$
\sum_{k \geq N}^{\infty}\left\|\left[\boldsymbol{u}_{k}, \boldsymbol{v}_{k}\right]^{T}-\left[\widetilde{\boldsymbol{u}}_{k}, \widetilde{\boldsymbol{v}}_{k}\right]^{T}\right\|_{\mathcal{H}}^{2}=\sum_{k \geq N}^{\infty} O\left(k^{-2}\right)<\infty .
$$


The same is true for their conjugates. Hence all conditions of Theorem 3 are satisfied. The result follows.

\section{EXPONEnTial STABILITY FOR SYSTEM (4.1)-(4.4)}

Theorem 5. Under the condition of Theorem 4, system (4.1)-(4.4) is exponentially stable. That is, there exist positive $M$ and $\omega$ such that the $C_{0}$-semigroup $e^{\mathcal{A t}}$ generated by $\mathcal{A}$ satisfies the inequality

$$
\left\|e^{\mathcal{A} t}\right\|_{\mathcal{H}} \leq M e^{-\omega t}
$$

Proof. We write further (4.17) as

$$
\operatorname{det}\left[M_{1}^{\prime}, M_{2}^{\prime}, M_{3}, M_{4}^{\prime}\right]=\operatorname{det} M^{\prime}+a \rho^{-1}+O\left(\rho^{-2}\right) .
$$

After a long but straightforward computation, we find that

$$
a=\frac{1}{4 \omega_{1}} \sum_{j=1}^{n-1}\left[i\left(p_{j}^{2}+q_{j}^{2}\right)+r_{j}+s_{j}\right]\left(e^{\rho \omega_{1} L_{j}}-e^{-\rho \omega_{1} L_{j}}\right)^{2} .
$$

Hence (4.22) can be written as

$$
e^{2 \omega_{1} \rho L_{n}}+1+\frac{1}{4 \rho \omega_{1}} \sum_{j=1}^{n-1}\left[i\left(p_{j}^{2}+q_{j}^{2}\right)+r_{j}+s_{j}\right]\left(e^{\rho \omega_{1} L_{j}}-e^{-\rho \omega_{1} L_{j}}\right)^{2}+O\left(\rho^{-2}\right)=0 .
$$

Substituting $\rho_{k}=-\frac{(k+1 / 2) \pi \omega_{1}}{L_{n}}+O\left(k^{-1}\right)$ into (6.1) and comparing the order of both sides as in [7], we can obtain the following asymptotic expressions of eigenvalues:

$$
\begin{aligned}
\rho_{k} & =\frac{(k+1 / 2) \pi \omega_{1}}{L_{n}}-\frac{1}{8(k+1 / 2) \pi \omega_{1}} \\
& \times \sum_{j=1}^{n-1}\left[p_{j}^{2}+q_{j}^{2}-i\left(r_{j}+s_{j}\right)\right] \sin ^{2} \frac{(k+1 / 2) \pi L_{j}}{L_{n}}+O\left(k^{-2}\right), \\
\lambda_{k} & =\rho_{k}^{2}=-\frac{1}{4 L_{n}} \sum_{j=1}^{n-1}\left(p_{j}^{2}+q_{j}^{2}\right) \sin ^{2} \frac{(k+1 / 2) \pi L_{j}}{L_{n}} \\
& \pm\left[\frac{(n+1 / 2)^{2} \pi^{2}}{L_{n}^{2}}+\frac{1}{4 L_{n}} \sum_{j=1}^{n-1}\left(r_{j}+s_{j}\right) \sin ^{2} \frac{(k+1 / 2) \pi L_{j}}{L_{n}}\right] i \\
& +O\left(k^{-1}\right), \quad k \rightarrow \infty .
\end{aligned}
$$

Finally, since system (4.1)-(4.4) is dissipative, it is easily shown that there is no $\lambda \in \sigma(\mathcal{A})$ such that $\operatorname{Re} \lambda=0$. Therefore, $\mathcal{A}$ generates an asymptotically stable $C_{0}$-semigroup on $\mathcal{H}$. By $(6.2)$, we see that this $C_{0}$-semigroup is also exponentially stable due to the spectrum-determined growth condition. The proof is complete. 


\section{Additional Remarks}

It is known that for the first-order homogeneous hyperbolic system (1.1), an equivalent new norm can be introduced for the state Hilbert space so that the system be dissipative under the new norm (see [14]) and hence the well-posedness is easily established by the Lumer-Phillips theorem in semigroup theory of linear operators [1]. However, such method seems not applicable to our system (1.2). Thus, the well-posedness for the general system (1.2) is still an unsolved problem. Our results in Sec. 5 show that in some cases, Riesz basis generation can be valid. But even for some cases of $n=1$, we do not know whether system (1.2) is a Riesz spectral system [12]. To explain this, let

$$
A=\left[\begin{array}{ll}
0 & 0 \\
1 & 1
\end{array}\right], \quad B=\left[\begin{array}{ll}
1 & 1 \\
0 & 0
\end{array}\right], \quad E=\left[\begin{array}{ll}
0 & 0 \\
1 & 0
\end{array}\right], \quad F=\left[\begin{array}{cc}
1 & -1 \\
0 & 0
\end{array}\right]
$$

in (1.2). Then this system is equivalent to the following system:

$$
\left\{\begin{array}{l}
y_{t t}(x, t)+y_{x x x x}(x, t)=0, \quad 0<x<1, \quad t>0, \\
y(0, t)=y_{x}(0, t)=y_{x x}(1, t)=0, \\
y_{x x x}(1, t)=y_{x t}(1, t),
\end{array}\right.
$$

which was studied in [18]. It was shown that this system is associated with an exponential stable 1-time integrated semigroup. In this case, $\operatorname{det} H(\lambda)=$ 0 reduces to

$$
\cosh (\tau) \cos (\tau)+i \sinh (\tau) \sin (\tau)+1=0
$$

and the eigenvalues $\lambda_{n}=i \tau_{n}^{2}$ can be found explicitly as

$$
\tau_{n}=(i+1)(n+1 / 2) \pi, \quad n=0,1, \ldots
$$

Moreover, each eigenvalue has the algebraic multiplicity 2 and the corresponding generalized eigenfunctions can be found as

$$
\begin{aligned}
{\left[\begin{array}{l}
u_{n} \\
v_{n}
\end{array}\right] } & =\cosh \left(\tau_{n} x\right)\left[\begin{array}{l}
1 \\
i
\end{array}\right]-i \cos \left(\tau_{n} x\right)\left[\begin{array}{c}
1 \\
-i
\end{array}\right], \\
{\left[\begin{array}{l}
u_{1 n} \\
v_{1 n}
\end{array}\right] } & =(x-i) \sinh \left(\tau_{n} x\right)\left[\begin{array}{l}
1 \\
i
\end{array}\right]+i(x+i) \sin \left(\tau_{n} x\right)\left[\begin{array}{c}
1 \\
-i
\end{array}\right] .
\end{aligned}
$$

However, we still do not know if there is a $C_{0}$-semigroup associated with (7.1) although we have explicit expressions of generalized eigenfunctions.

\section{Appendix: Proof of Lemma 2}

Since characteristic equation (4.22) of system (4.1)-(4.4) possesses only simple roots for $\rho$ with sufficiently large modulus, we can obtain corresponding eigenfunctions by calculating the determinant of the matrix $\widetilde{M}_{j}$ which is obtained by replacing one of the rows of $\tilde{M}$ in (4.7) by $u_{j}$ or $v_{j}, j=1, \ldots, n$, in (3.11), such that $\operatorname{det} \widetilde{M}_{j} \neq 0$. Fix $j, 1 \leq j \leq n$, and substitute $u_{j}$ into 
$(2 n+j)$ th row of $\tilde{M}$ and then multiply $\operatorname{det} \widetilde{M}_{j}$ by $e^{\left(\omega_{1}+\omega_{2}\right) \rho L_{n}}$. After setting all terms containing $e^{\omega_{2} \rho l_{s}}$ for some $s$ to be zero and taking dominant terms with respect to the order of $\rho$ and exchanging lines and rows in this matrix, we obtain

$$
u_{j}(x)=\operatorname{det} \widetilde{M}_{j}=e^{-\left(\omega_{1}+\omega_{2}\right) \rho L_{n}} \cdot\left[\operatorname{det} \widetilde{M}_{j}^{\prime}+O\left(\rho^{-1}\right)\right],
$$

where $\widetilde{M}_{j}^{\prime}$ has the same form as $M^{\prime}$ in (4.18) but with $N_{j 1}$ and $N_{j 2}$ replaced by $\widetilde{N}_{j 1}$ and $\widetilde{N}_{j 2}$ of the following form:

$$
\begin{aligned}
& \widetilde{N}_{0}=N_{0}, \\
& \widetilde{N}_{n}=\left[\begin{array}{cccc}
(1-i) e^{\omega_{1} \rho l_{n}} & 0 & -1+i & 1-i \\
e^{\omega_{1} \rho l_{n} x} & e^{\omega_{2} \rho l_{n} x} & e^{\omega_{1} \rho l_{n}(1-x)} & e^{\omega_{2} \rho l_{n}(1-x)}
\end{array}\right] \text {, }
\end{aligned}
$$

and

$$
\begin{aligned}
\widetilde{N}_{j 1} & =\left[\begin{array}{llll}
\widetilde{Q}_{j 1}, & \widetilde{Q}_{j 2}, & \widetilde{Q}_{j 3}, & \widetilde{Q}_{j 4}
\end{array}\right], \\
\widetilde{N}_{j 2} & =\left[\begin{array}{llll}
\widetilde{R}_{j 1}, & \widetilde{R}_{j 2}, & \widetilde{R}_{j 3}, & \widetilde{R}_{j 4}
\end{array}\right],
\end{aligned}
$$

where for odd $j$,

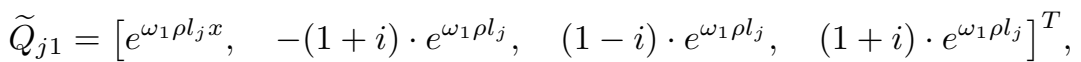

$$
\begin{aligned}
& \widetilde{Q}_{j 2}=\left[\begin{array}{llll}
e^{\omega_{2} \rho l_{j} x}, & 0, & 0, & 0
\end{array}\right]^{T},
\end{aligned}
$$

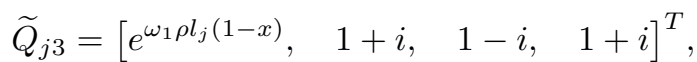

$$
\begin{aligned}
& \widetilde{Q}_{j 4}=b i g\left[e^{\omega_{2} \rho l_{j}(1-x)}, \quad 1+i, \quad 1+i, \quad 1-i\right]^{T}, \\
& \widetilde{R}_{j 1}=\left[\begin{array}{lll}
0, & (1-i) e^{\omega_{1} \rho l_{j+1}}, \quad-(1+i) e^{\omega_{1} \rho l_{j+1}}, \quad(1-i) e^{\omega_{1} \rho l_{j+1}}
\end{array}\right]^{T}, \\
& \widetilde{R}_{j 2}=\left[\begin{array}{llll}
0, & 0, & 0, & 0
\end{array}\right]^{T}, \\
& \widetilde{R}_{j 3}=\left[\begin{array}{lll}
0, & -(1-i), \quad-(1+i), \quad 1-i
\end{array}\right]^{T},
\end{aligned}
$$

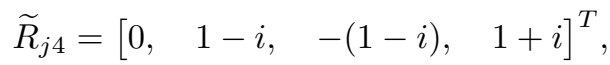

and for even $j$,

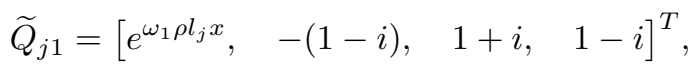

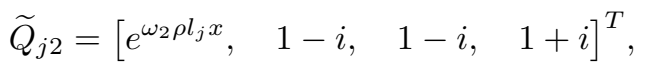

$$
\begin{aligned}
& \widetilde{Q}_{j 3}=\left[\begin{array}{llll}
e^{\omega_{1} \rho l_{j}(1-x)}, & (1-i) e^{\omega_{1} \rho l_{j}}, \quad(1+i) e^{\omega_{1} \rho l_{j}}, \quad(1-i) e^{\omega_{1} \rho l_{j}}
\end{array}\right]^{T}, \\
& \widetilde{Q}_{j 4}=\left[e^{\omega_{2} \rho l_{j}(1-x)}, \quad 0, \quad 0, \quad 0\right]^{T} \text {, }
\end{aligned}
$$

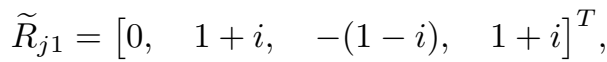

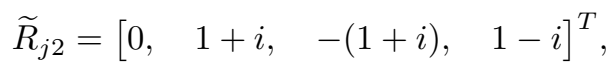

$$
\begin{aligned}
& \widetilde{R}_{j 3}=\left[\begin{array}{lll}
0, & -(1+i) e^{\omega_{1} \rho l_{j+1}}, \quad-(1-i) e^{\omega_{1} \rho l_{j+1}}, \quad(1+i) e^{\omega_{1} \rho l_{j+1}}
\end{array}\right]^{T},
\end{aligned}
$$




$$
\widetilde{R}_{j 4}=\left[\begin{array}{llll}
0, & 0, & 0, & 0
\end{array}\right]^{T} .
$$

Let us consider only odd $j \neq n$, since other cases could be treated similarly. We can obtain (cf. (4.19))

$$
\operatorname{det} \widetilde{M}_{j}^{\prime}=(-1)^{(n+1) / 2} \cdot 16^{n} \cdot \frac{i}{2} \cdot \operatorname{det} \widetilde{S}_{j},
$$

where like $S$ in (4.19), $\widetilde{S}_{j}$ is a $(2 n \times 2 n)$-matrix and can be obtained from $S$ with $N_{j 1}^{\prime}$ and $N_{j 2}^{\prime}$ replaced by $\widetilde{N}_{j 1}^{\prime}$ and $\widetilde{N}_{j 2}^{\prime}$ of the following form:

$$
\begin{aligned}
\tilde{N}_{j 1}^{\prime} & =\left[\begin{array}{cc}
\alpha_{j} & \beta_{j} \\
(1-i) e^{\omega_{1} \rho l_{j}} & 1-i
\end{array}\right], \\
\tilde{N}_{j 2}^{\prime} & =\left[\begin{array}{cc}
-\frac{1-i}{2} e^{\omega_{1} \rho l_{j+1}} \cdot e^{\omega_{2} \rho l_{j}(1-x)} & -\frac{1+i}{2} e^{\omega_{2} \rho l_{j}(1-x)} \\
-(1+i) e^{\omega_{1} \rho l_{j+1}} & -1-i
\end{array}\right],
\end{aligned}
$$

where

$$
\begin{aligned}
& \alpha_{j}=e^{\omega_{1} \rho l_{j} x}+i e^{\omega_{2} \rho l_{j} x}+\frac{1-i}{2} e^{\omega_{1} \rho l_{j}} \cdot e^{\omega_{2} \rho l_{j}(1-x)}, \\
& \beta_{j}=e^{\omega_{1} \rho l_{j}(1-x)}+i e^{\omega_{1} \rho l_{j}} \cdot e^{\omega_{2} \rho l_{j} x}-\frac{1+i}{2} e^{\omega_{2} \rho l_{j}(1-x)} .
\end{aligned}
$$

Next, similarly to (4.20), we have

$$
\operatorname{det} \widetilde{S}_{j}=4^{n-2} \cdot S_{0} \cdot S_{1} \cdots S_{j-1} \cdot \widehat{S}_{j} \cdot S_{j+1} \cdots S_{n},
$$

where $S_{l}, l=0,1, \ldots, n, l \neq j$, are given by (4.21) and $\widehat{S}_{j}$ is given by

$$
\widehat{S}_{j}=\left[\begin{array}{ll}
\widehat{S}_{j 1} & \widehat{S}_{j 2} \\
\widehat{S}_{j 3} & \widehat{S}_{j 4}
\end{array}\right],
$$

where

$$
\begin{aligned}
\widehat{S}_{j 1}=- & (1+i) e^{\omega_{1} \rho\left(l_{j}+l_{j+1}-l_{j} x\right)}+(1-i) e^{\omega_{1} \rho\left(l_{j}+l_{j+1}\right)} \cdot e^{\omega_{2} \rho l_{j} x}, \\
\widehat{S}_{j 2}=- & (1+i) e^{\omega_{1} \rho l_{j}(1-x)}+(1-i) e^{\omega_{1} \rho l_{j}} \cdot e^{\omega_{2} \rho l_{j} x} \\
& +(1+i) e^{\omega_{2} \rho l_{j}(1-x)} \\
\widehat{S}_{j 3}= & (1+i) e^{\omega_{1} \rho\left(l_{j}+l_{j+1}\right)} \cdot e^{\omega_{2} \rho l_{j}(1-x)}+(1+i) e^{\omega_{1} \rho\left(l_{j+1}+l_{j} x\right)} \\
& \quad-(1-i) e^{\omega_{1} \rho l_{j+1}} \cdot e^{\omega_{2} \rho l_{j} x} \\
\widehat{S}_{j 3}= & (1+i) e^{\omega_{1} \rho l_{j} x}-(1-i) e^{\omega_{2} \rho l_{j} x} .
\end{aligned}
$$

Substituting (8.5) and (8.6) into (8.4) gives

$$
\begin{aligned}
\operatorname{det} \widetilde{S}_{j} & =4^{n-2}(1+i) \cdot\left(e^{\omega_{1} \rho L_{j}}-e^{-\omega_{1} \rho L_{j}}\right) \cdot\left[e^{\omega_{1} \rho\left(L_{j-1}+l_{j} x\right)}\right. \\
& \left.-e^{-\omega_{1} \rho\left(L_{j-1}+l_{j} x\right)}+i e^{\omega_{2} \rho l_{j} x} \cdot\left(e^{\omega_{1} \rho L_{j-1}}-e^{-\omega_{1} \rho L_{j-1}}\right)\right] .
\end{aligned}
$$


For either even $j$ or $j=n$, we always have

$$
\begin{aligned}
\operatorname{det} \widetilde{S}_{j} & =4^{n-2}(1-i) \cdot\left(e^{\omega_{1} \rho L_{j}}-e^{-\omega_{1} \rho L_{j}}\right) \cdot\left[e^{\omega_{1} \rho\left(L_{j}-l_{j} x\right)}\right. \\
& \left.-e^{-\omega_{1} \rho\left(L_{j}-l_{j} x\right)}-i e^{\omega_{2} \rho l_{j}(1-x)} \cdot\left(e^{\omega_{1} \rho L_{j-1}}-e^{-\omega_{1} \rho L_{j-1}}\right)\right], \\
& j=2,4, \ldots, n-1, \\
\operatorname{det} \widetilde{S}_{n} & =-4^{n-1} \cdot e^{\omega_{1} \rho L_{n}} \cdot\left[e^{\omega_{1} \rho\left(L_{n-1}+l_{n} x\right)}-e^{-\omega_{1} \rho\left(L_{n-1}+l_{n} x\right)}\right. \\
& \left.+i e^{\omega_{2} \rho l_{n} x} \cdot\left(e^{\omega_{1} \rho L_{n-1}}-e^{-\omega_{1} \rho L_{n-1}}\right)\right] .
\end{aligned}
$$

Combining (8.1), (8.2), and (8.7)-(8.9), we finally obtain (up to a nonzero scalar) (4.23)-(4.26). The proof is complete.

Acknowledgment. The authors would like to thank the anonymous referee and Dr. Runyi Yu of Eastern Mediterranean University for their valuable comments and suggestions. The support of the National Natural Science Foundation of China is gratefully acknowledged.

\section{REFERENCES}

1. A. Pazy, Semigroups of linear operators and applications to partial differential equations. Springer-Verlag, Berlin (1983).

2. G. Chen, M. C. Delfour, A. M. Krall, and G. Payre, Modeling, stabilization, and control of serially connected beam. SIAM J. Control Optim. 25 (1987), 526-546.

3. G. Chen, S. G. Krantz, C. E. Wayne, H. H. West, and M. P. Coleman, Analysis, design, and behavior of dissipative joints for connected beams. SIAM J. Appl. Math. 49 (1989), 1665-1693.

4. G. Chen and J. Zhou, Vibration and damping in distributed systems, Vols. I, II. Stud. Adv. Math. (1993).

5. G. Chen, S. G. Krantz, D. W. Ma, C. E. Wayne, and H. H. West, The Euler-Bernoulli beam equation with boundary energy dissipation. In: Proc. Operator Methods for Optimal Control Problems (S. J. Lee, ed.) Lect. Notes Pure Appl. Math. No. 108, Marcel Dekker, New York (1988), pp. 67-96.

6. F. Conrad and O. Mörgul, On the stabilization of a flexible beam with a tip mass. SIAM J. Control Optim. 36 (1998), 1962-1986.

7. B. Z. Guo, Riesz basis approach to the stabilization of a flexible beam with a tip mass. SIAM J. Control Optim. 39 (2001), 1736-1747.

8. _ Riesz basis property and exponential stability of controlled Euler-Bernoulli beam equations with variable coefficients. SIAM J. Control Optim. 40 (2002), No. 6, 1905-1923.

9. B. Z. Guo and Y. H. Luo, Riesz basis property of a second order hyperbolic system with collocated scalar input/output. IEEE Trans. Automat. Control 47 (2002), 693-698. 
10. B. Z. Guo and K. Y. Chan, Riesz basis generation, eigenvalues distribution, and exponential stability for an Euler-Bernoulli beam with joint feedback control. Rev. Mat. Complut. 14 (2001), No. 1, 205-229.

11. L. F. Ho, Controllability and stabilizability of coupled strings with control applied at the coupled points. SIAM J. Control Optim. 31 (1993), 1416-1437.

12. R. F. Curtain and H. J. Zwart, An introduction to infinite-dimensional linear system theory. Springer-Verlag, New York (1995).

13. M. A. Naimark, Linear differential operators. Vol. I, Ungar, New York (1967).

14. A. F. Neves, H. De S. Riberiro, and O. Lopes, On the spectrum of evolution operators generated by hyperbolic systems. J. Funct. Anal. 67 (1986), 320-344.

15. J. L. Lions, Exact controllability, stabilization and perturbations for distributed systems. SIAM Rev. 30 (1988), 1-68.

16. K. S. Liu, Energy decay problems in the design of a point stabilizer for connected string vibrating systems. SIAM J. Control Optim. 26 (1988), $1348-1356$.

17. K. S. Liu, F. L. Huang, and G. Chen, Exponential stability analysis of a long chain of connected vibrating strings with dissipative linkage. SIAM J. Appl. Math. 49 (1989), 1694-1707.

18. Z. H. Luo and B. Z. Guo, Shear force feedback control of a single-link flexible robot with a revolute joint. IEEE Trans. Automat. Control 42 (1997), 53-65.

19. Z. H. Luo, B. Z. Guo, and O. Mörgul, Stability and stabilization of infinite-dimensional system with applications. Springer-Verlag, London (1999).

20. S. G. Krantz and W. H. Paulsen, Asymptotic eigenfrequency distributions for the $N$-beam Euler-Bernoulli connected beam equation with dissipative joints. J. Symbolic Comput. 11 (1991), 369-418.

21. R. Rebarber, Exponential stability of connectedbeams with dissipative joints: a frequency domain approach. SIAM J. Control Optim. 33 (1995), No. 1, 1-28.

22. M. Renardy, On the linear stability of hyperbolic PDEs and viscoelastic flows. Z. Angew. Math. Phys. 45 (1994), 854-865.

23. G. Q. Xu and B. Z. Guo, Riesz basis property of evolution equations in Hilbert spaces and application to a coupled string equation. SIAM J. Control Optim. 42 (2003), No. 3, 966-984. 
Authors' addresses:

Bao-Zhu Guo

Institute of Systems Science,

Academy of Mathematics and System Sciences

Academia Sinica, Beijing 100080, China

E-mail: bzguo@mail.iss.ac.cn

$\mathrm{Yu} \mathrm{Xie}$

Institute of Systems Science,

Academy of Mathematics and System Sciences

Academia Sinica, Beijing 100080, China

Xue-Zhang Hou

Mathematics Department

Towson University

8000 York Road, Towson, Maryland 21252, USA 